\title{
Bioabsorbable Vascular Scaffold: A Revolution in Coronary Intervention
}

\author{
M Ullah, AKMM Islam, AAS Majumder \\ Department of Cardiology, NICVD, Dhaka
}

\begin{abstract}
Key Words : PCI, Bioabsorbable vascular stents, Coronary artery disease.

Since the advent of percutaneous coronary angioplasty in 1977, immense experiment has been done to improve the outcome of the patients with coronary artery disease. Lot of trials have been done with angioplasty, bare metal stents, drug eluting stents, drug eluting balloons and other devices. Bioabsorbable vascular stents are relatively newcomers in this field with a lot of hope. We tried to update the latest status of Bioabsorbable vascular stents in this review, specially the short and midterm safety and efficacy and some of their limitations.
\end{abstract}

(Cardiovasc. j. 2014; 6(2): 149-163)

\section{Introduction:}

Coronary stents have become the default device in percutaneous coronary interventions (PCIs). They are used as a mechanical means to overcome the major limitations of balloon angioplasty - early recoil and late vascular remodeling. ${ }^{1}$ The major limitations of stents are thrombosis and restenosis. Drug-eluting stents (DES) have reduced restenosis rates and the need for repeat revascularization, however stent thrombosis, although controlled with antiplatelet therapy, remains a limitation of DES. Nevertheless, the role of stenting is temporary and is limited to the period of intervention and shortly thereafter, until healing and reendothelialization are obtained. Beyond that, no utility or advantage for stents has been demonstrated and their presence could be a nidus for late thrombosis and chronic inflammation. Considering the complications of both bare metal stents and DES, question arose: "Why is a permanent prosthesis needed to fix a temporary problem?"

For this reason a disappearing stent is searched for long time which will act as a scaffold only for the essential period of time after PCI. This is named as bioabsorbable stent, bioresorbable stent, bioresorbable vascular scaffold (BVS), bioresorbable vascular scaffolding device or disappearing stent in different literatures. When assessing bioresorbable scaffold (BRS) technology, there are important questions to be asked:

- How long a scaffold is essential for the prevention of vessel recoil?
- What is the optimal rate of degradation of scaffold material? Does it completely degrade? If so, at what time points?

- What happens to the degraded products?

- What is the vessel composition following complete degradation?

- Are there any safety concerns?

- Are there any potential utility advantages of BRS over permanent metal stents? Will BRS ever be the workhorse stent to replace bestin-class DES?

First bioabsorbable stent, the Igaki-Tamai stent was implanted in human coronary arteries in 1999. Since then a number of bioabsorbable stent has been tried. There are challenges in making a stent that has sufficient radial strength for an appropriate duration, which does not have unduly thick struts, which can be a drug delivery vehicle, and where degradation does not generate an unacceptable inflammatory response. We will review different bioabsorbable stents those have been studied clinically.

\section{Why a biodegradable stent is needed:}

From the advent of the technology in 1977, different ways and devices were tried to open and maintain the patency of stenosed coronary arteries. But every procedure and device (balloon angioplasty, bare metal stent, drug eluting stent) has got their advantages and drawbacks. 


\begin{tabular}{|c|c|c|c|}
\hline & $\begin{array}{l}1977 \\
\text { Balloon Angioplasty }\end{array}$ & $\begin{array}{r}1987 \\
\text { Bare Metal Stent }\end{array}$ & $\begin{array}{c}2000 \\
\text { Drug Eluting Stent }\end{array}$ \\
\hline Advantages & $\begin{array}{l}{ }^{*} \text { Reduces the } \\
\text { atherosclerotic blockage. } \\
\text { *Less focal injury. } \\
\text { *Temporary strain. }\end{array}$ & $\begin{array}{l}\text { *Prevents elastic recoil. } \\
\text { *Prevents vascular } \\
\text { remodeling. }\end{array}$ & $\begin{array}{l}\text { *Anti inflammatory. } \\
\text { *Anti platelet. } \\
\text { *Antiproliferative. } \\
\text { *Antimigratory. }\end{array}$ \\
\hline Limitations & $\begin{array}{l}\text { *Elastic recoil. } \\
\text { *Thrombus formation. } \\
\text { * Inflamation leading to } \\
\text { restenosis. }\end{array}$ & $\begin{array}{l}\text { *Deep focal injury. } \\
\text { *Inflamation leading to } \\
\text { restenosis. } \\
\text { * Subacute thrombosis. }\end{array}$ & $\begin{array}{l}\text { *Delays the formation of } \\
\text { healthy endothelium. } \\
\text { *Late stent thrombosis. } \\
\text { *Longer term antiplatelet } \\
\text { therapy. }\end{array}$ \\
\hline
\end{tabular}

The motivation for the development of bioabsorbable stents was driven by the need to solve the limitations of metallic stents, such as stent thrombosis, which requires prolonged antiplatelet therapy along with the risk of bleeding; mismatch of the stent to the vessel size, which often results in a smaller lumen after stent implantation; and artifacts with modern imaging technologies such as magnetic resonance imaging (MRI) and multi-slice computerized tomography (MSCT). Other potential limitations of metallic stents are impairment of vessel geometry and often jailing of side braches. Further, metallic stents prevent the lumen expansion associated with late favorable remodeling and vessel reactivity at the stented site. $^{2}$ Finally, excessive use of stents in the coronary vasculature (full metal jacket) may interfere with traditional re-interventional techniques such as bypass graft surgery.

These lead to the necessity of development of bioabsorbable stents that will leave behind only the healed, natural vessel allowing restoration of vasoreactivity with the potential for vessel remodeling. Because of their ability to exist for a short period of time and healing the diseased, stenosed vessel to a normal one, PCI with bioabsorbable stent is also termed as vascular scaffold therapy (VST) or vascular reparative therapy (VRT).

\section{Goals of VRT:}

If with advancement of technology proper bioabsorbable stents are made available many disadvantages of metallic stents can be avoided.
Unlike with permanent metallic stenting, the BVS will potentially allow future surgical revascularization, expansive remodeling, restoration of reactive vasomotion, and noninvasive imaging of coronary arteries with multislice computed tomography (MSCT). Given their temporary presence, the potential risk of late or very late stent thrombosis may be reduced or even eliminated.

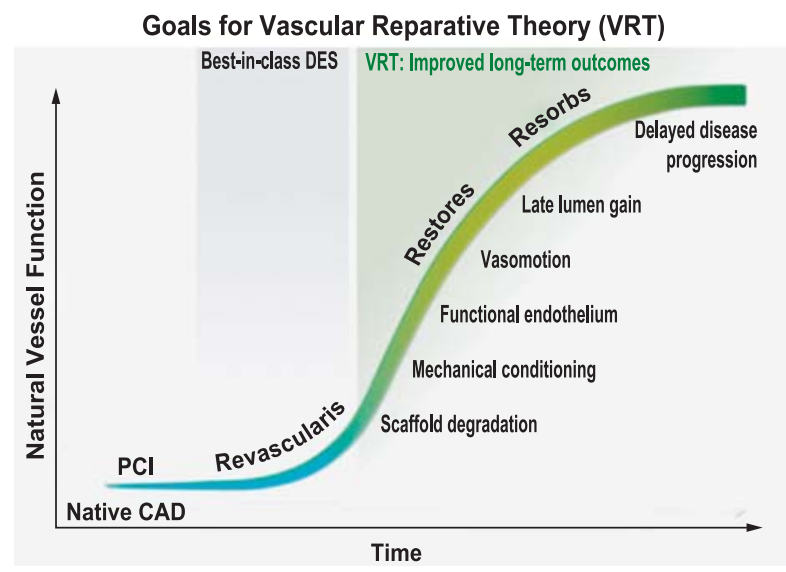

Fig.-1: Goal of vascular reparative therapy (Reprinted from http://www.abbottvascular. com/int/absorb.html\#features).

\section{Challenges of biodegradable stents:}

The manufacturing of biodegradable stents is a challenging job due to following reasons-

1. The available polymers with adequate radial force and scaffolding ability like a metallic stent are few in number.

2. The degradation time and degradation rate of stent material should be adequate for 
prevention of acute and late recoil of blood vessels.

3. Most of the biodegradable materials available are radiolucent, so not visible by angiography. So their proper implantation needs further imaging technique like optical coherence tomography (OCT), which needs more hardware and expertise.

4. To prevent neoinitimal growth thus restenosis anti proliferative agents should be added to it. That means another vehicle material should be added to it. The degradation rate and the elution rate of drugs should be matched perfectly to prevent any untoward effects.

5. The end products of the polymer and the metal need to be addressed regarding whether they will reabsorb over time without creating systemic or local vessel toxicity and the preservation of the biocompatability with endothelial coverage and function.

\section{How long vascular scaffold is needed}

Stents scaffold intimal flaps that have been separated from deeper layers during angioplasty, prevent early constrictive remodeling, and deliver antiproliferative drug to limit excessive healing of injured vessel. This support is essential during the initial months after the procedure. After this, a permanent implant is unnecessary and has potential disadvantages.

But how long a stent is needed to prevent vascular recoil and constrictive remodeling is not clearly defined. In a cohort of patients consecutively recatheterized at 1, 2, 3, and 4 months, Serruys et $\mathrm{al}^{3}$ demonstrated that the restenotic process after balloon angioplasty ceases to progress after 4 months. It is possible that after this time, scaffolding is no longer needed.

The poly-L-Lactide acid (PLLA) made non-drugeluting Igaki-Tamai device and drug eluting ABSORB stent did not show bioresorption of the polymeric struts at 6 months and this absence of ultrasonic changes in struts was parallel to the absence of scaffold shrinkage. This device presented a target vessel revascularization of $6.7 \%$ at 6 months and a low rate of major cardiac events at 10 years. ${ }^{4,5}$ Conversely, the PROGRESS-AMS magnesium platform non- drug-eluting bioresorbable scaffold had a rapid resorption which was complete at 4 -month follow-up. This swift resorption produced an important reduction of the lumen $(60 \%$ of the late lumen loss) and a high incidence of restenosis (47.5\% assessed by quantitative coronary angiography (QCA). ${ }^{6}$ The REVA device is a poly (iodinated desaminotyrosyltyrosine ethyl ester) carbonate non-drug-eluting scaffold. The closed design and the lifecycle of the carbonate provide enough radial strength during the first 3 months following the implantation without appreciable shrinkage. However, focal mechanical failures driven by polymer embrittlement led to a high rate of target lesion revascularization (TLR) (66.7\%) between 4 - and 6-month follow-up. ${ }^{7}$

ABSORB 1.0, ABSORB 1.1 and the Magnesium stent used in BIOSOLVE- I trial provided their scaffold function for at least 6 months. Though they are drug eluting stents their structural integrity upto six months reduced the late recoil and constrictive remodeling of blood vessel. Even the slower degradation of ABSORB 1.1 over ABSORB 1.0 was also of benefit in prevention of late recoil.

So for any bioabsorbable stent it should provide a good mechanical support for 6 months and then disappear.

\section{Materials for bioabsorbable stent}

Currently, there are number of materials being tried for bioabsorbable scaffolds, of which lactide polymers, particularly PLLA, form the basis of several devices and are the most extensively investigated. Other materials include magnesium, polyanhydrides (salicylic acid and adipic acid) and polycarbonates (amino acids, eg, tyrosine).

\section{Life cycle of PLLA}

The poly-L-lactic acid (PLLA) has a lifecycle which can be divided in five phases. ${ }^{8}$ First, immediately after the deployment, the polymer absorbs water from blood and surrounding tissues. Second, the long chains of PLLA degrade by hydrolysis into smaller chains without affecting the device's structure. Third, the hydrolysis process continues and causes a loss in integrity, with fragmentation of the struts and loss of radial strength. Fourth, soluble monomeric anions dissolve into the intercellular fluid and microparticles of less than $2 \mathrm{~mm}$ may 
be phagocytosed by the macrophages; manifesting in mass loss and bioresorption. Finally, the soluble L-lactate is converted into pyruvate, which enters the Krebs cycle, being eventually converted into carbon dioxide and water. The initial degradation process of the PLLA semi-crystalline polymer depends on the length of the polymers chain (molecular weight), the hydrophilicity, and the degree of crystallinitiy. The duration of the degradation process varies from 2 to 4 years.
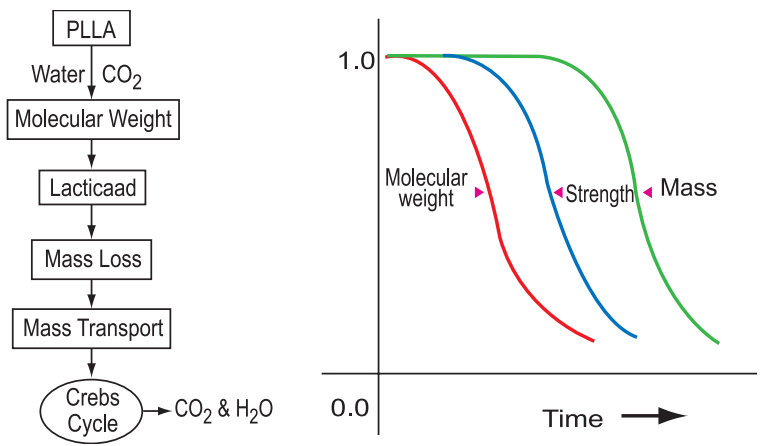

Fig.-2: (Left) The metabolism of poly-L-lactic acid (PLLA) biodegradable stents. Hydrolysis of PLLA results in the loss of molecular weight, and reduction in strength and mass; ultimately the PLLA is metabolized into lactic acid, carbon dioxide (CO2) and water (H2O). (Right) Bioabsorption curves for a bioabsorbable material: molecular weight is lost first, followed by strength and then mass. Therefore, the stent loses its biomedical importance long before significant mass loss has occurred. ${ }^{9}$ (Reproduced with permission.)

\section{Improvement in PLLA based Bioabsorbable Vascular Scaffold (BVS) design-}

The most common PLLA based bioabsorbable stent is ABSORB stent. ABSORB 1.0 (Abbott Vascular, Santa Clara, CA, USA) is built on a backbone of semi-crystalline poly-L-lactide (PLLA) polymer. The polymer consists of crystalline and amorphous domains. The platform is coated with the poly-D,L-lactide (PDLLA) copolymer that contains and controls the release of the antiproliferative everolimus (Novartis, Switzerland). Both PLLA and PDLLA are fully bioresorbable. The strut thickness is $150 \mu \mathrm{m}$ and the struts are distributed as circumferential out- of-phase zigzag hoops linked together by three longitudinal bridges between each hoop. The BVS 1.0 design is shown in Figure 3. In ABSORB clinical trial (cohort A), despite the impressive clinical outcomes, that were comparable with the XIENCE V ${ }^{\circledR}$ everolimus eluting stent (Abbott Vascular, Santa Clara, CA, USA), the ABSORB 1.0 device had a slightly higher, but insignificant acute and late recoil. There was a trend for more recoil when fewer struts were present. These observations prompted design modifications in order to reduce the maximum circular unsupported cross sectional area (MCUSA), increase radial force, and prolong the time for which the implant scaffolds the vessel, without changing the implant's total absorption time.

The manufacturing process of the ABSORB 1.1 (Abbott Vascular, Santa Clara, CA, USA) has been modified to enhance the mechanical strength and mechanical durability of the struts. Moreover, the new design has in-phase zigzag hoops linked by bridges that allow for a more uniform strut distribution, reduce MCUSA, and provide more uniform vessel wall support and drug transfer. ${ }^{10}$ The polymer mass, coating content, amount of drug, and the strut thickness remain the same. This change in design leads to slower degradation of the scaffold and slower release of drugs. An OCT ad hoc analysis of the revision 1.0 and revision 1.1 stent design in the ABSORB clinical trial by Okamura et al showed that the new design has a reduced maximum circular unsupported cross sectional area. This change in design resulted in reduced late shrinkage and neointimal response with BVS 1.1. ${ }^{10}$ The actual duration of resorption of the second generation in vivo is approximately 18 months longer than that of the first generation, and the mass loss of the second-generation ABSORB scaffold takes approximately 36 months.

At 6-month follow-up, patients treated with the ABSORB 1.0 had a significant decrease in minimum luminal diameter (MLD) (angiographic late loss) of $0.43 \mathrm{~mm}(\mathrm{p}=0.01)$, whereas a nonsignificant $0.08 \mathrm{~mm}$ decrease was seen in those treated with the BVS 1.1. The difference in late loss between the BVS 1.0 and 1.1, although numerically appreciable, failed to reach statistical significance at 6 months $(\mathrm{P}=0.07) .{ }^{11}$ 
In another study comparing two generation of stents by Serruys et al showed that with ABSORB 1.1 the late lumen loss amounted to $0.19 \pm 0.18 \mathrm{~mm}$ with a limited relative decrease in minimal luminal area of $5.4 \%$ on intravascular ultrasound (IVUS). OCT showed at follow-up that $96.8 \%$ of the struts were covered. They concluded that modified manufacturing process of the polymer and geometric changes in the polymeric platform have substantially improved the medium-term performance of this new generation of drug-eluting scaffold to become comparable to those of current drug eluting stents. ${ }^{12}$

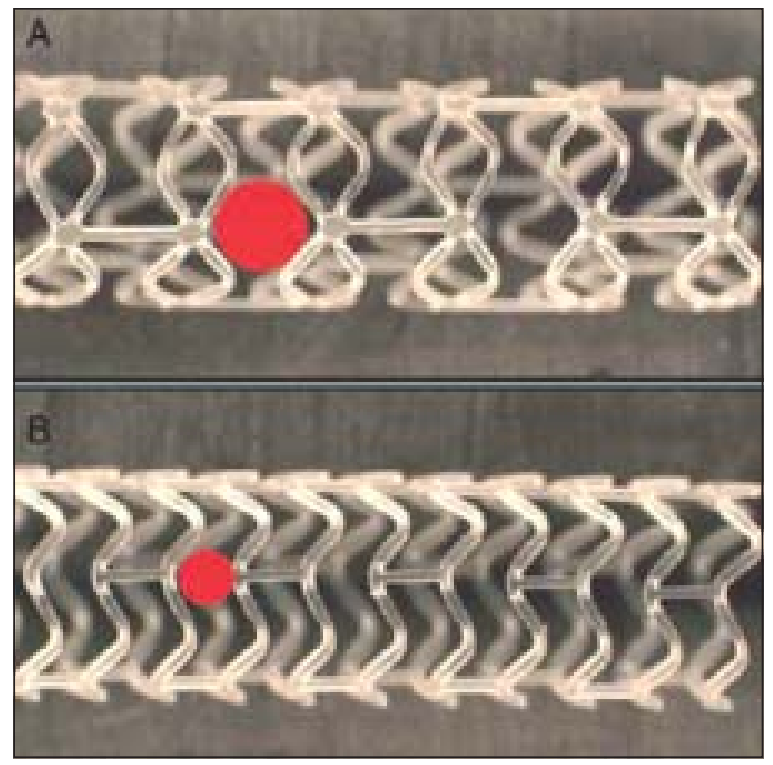

Fig.-3: Design of the different bioresorbable vascular scaffold (BVS). (A) BVS 1.0 design. The struts are distributed as circumferential out-ofphase zigzag hoops linked together by three longitudinal bridges between each hoop. The maximal circular unsupported surface area is drawn as a red circle. (B) BVS 1.1 design. The struts are arranged as in-phase zigzag hoops linked together by three longitudinal bridges. The strut distribution is more uniform and allows the maximal circular unsupported surface area (red circle) to be smaller than in the BVS 1.0. ${ }^{11}$ (Reproduced with permission.)

\section{Vascular compliance and BVS:}

From a mechanical perspective, stenting the coronary artery may locally stiffen the artery by reducing its compliance and creating a mismatch in compliance with respect to the segments contiguous to the implanted device. ${ }^{12}$ This mismatch may eventually provoke flow disturbances and wall shear stress alterations with subsequent blood stasis (Figure 4). ${ }^{14}$ The wall shear stress distribution in a stented artery has been reported as a determinant factor for cellular growth and the occurrence of thrombus formation. ${ }^{15}$

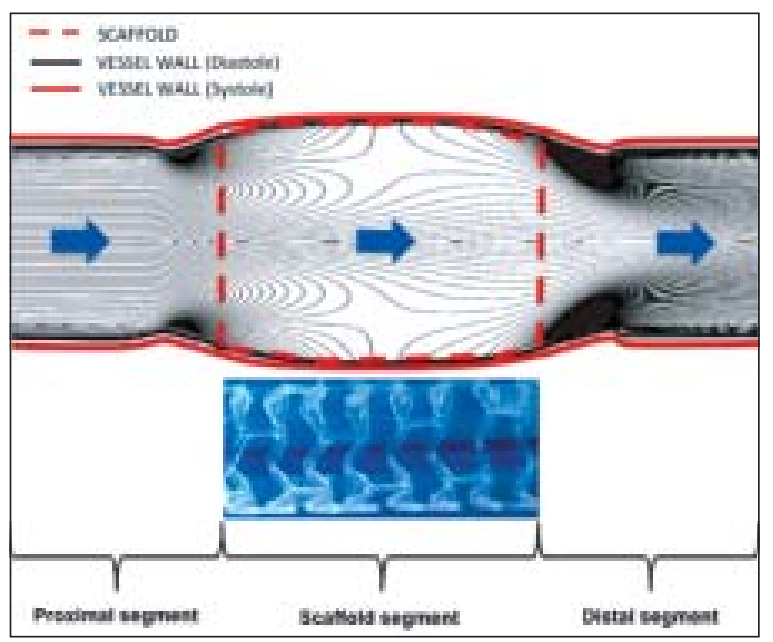

Fig.-4: Compliance mismatch after scaffold implantation with alteration in the flow. In this diagram the mismatch in compliance created by the scaffold (red dotted line) is indicated as a "bump" in the vessel wall compared to the proximal and distal segments. Instantaneous vortices fields calculated by a mathematical model are also shown, indicating presence of turbulence at the proximal and distal edges. ${ }^{16}$ (Reproduced with permission.)

The bioresorbable vascular scaffold system theoretically has many advantages over rigid metallic stents. In particular, because the scaffold is completely made of poly-lactide, it does not have the same stiffness as metal, thereby having the potential to overcome in part the problems related to local stiffening of the artery and compliance mismatch associated with metallic platform stents. In addition, the mismatch in compliance after scaffold implantation may potentially disappear in the long term once the scaffold is completely bioresorbed.In the study involving 83 patients of ABSORB trial, Brugaletta et al have shown that (1) scaffolding of a diseased vessel wall by an ABSORB BVS significantly reduces its compliance, with the compliance of the segment immediately distal to the device tending also to be reduced; (2) the mismatch in compliance that 
is present immediately after scaffold implantation disappears in the short- to midterm. ${ }^{16}$

\section{BVS and vascular geometry}

Stiff metallic stents can alter vessel geometry and biomechanics leading to long-term flow disturbances and chronic irritation producing adverse events. This change in vessel biomechanics is also responsible for late strut fractures, that could lead to restenosis and clinical events. ${ }^{17,18}$ After metallic stent implantation, the curvature of target vessel is increased by $121 \%$ at the entrance and by $100 \%$ at the exit of the stent, resulting in local changes in shear stress correlated with the local curvature. The initial superior conformability and flexibility of the ABSORB with respect to metallic stents can, at an early stage, contribute to less change in vessel geometry and biomechanics. ${ }^{18}$

A retrospective study compares 102 patients who received a metallic stent (Multi-link Vision or Xience V) in the SPIRIT FIRST and II trials with 89 patients treated with the ABSORB Revision 1.1 everolimus-eluting BVS from cohort B of the ABSORB trial. Both the metallic stent (MPS) and BVS groups had significant changes in relative region curvature (MPS vs.BVS: $28.7 \%$ vs. $7.5 \%$ ) and angulation (MPS vs. BVS: $25.4 \%$ vs. $13.4 \%$ ) after deployment. The unadjusted comparisons between the 2 groups showed, for BVS a nonsignificant trend for less change in region curvature after deployment and a significantly lower modification of angulation. By multivariate regression analysis, the independent predictors of changes in curvature and angulation were the pretreatment region curvature, the pre-treatment region angulation, and the used device. ${ }^{18}$ At 6 - or 12 -month followup, the anatomical configuration of the arteries treated with the ABSORB BVS allowed restoration of coronary geometry to values close to those measured before implantation. ${ }^{19}$

\section{Late vascular remodeling in metallic stent and BVS}

One possible fate of the dilated but caged lesion is an intra-stent lumen reduction by intra-stent neointimal tissue growth (negative remodeling), even if the cytostatic drug slows down or postpones the phenomenon. But in many of the cases there is retraction of the surrounding vessel wall from the metallic cage. This generates a late acquired malapposition. It has been demonstrated that a large malapposition at baseline will also persist at long term. Late and very late stent thrombosis has been associated with late malapposition, either acquired or persistent. ${ }^{20}$ But in case of BVS, as the scaffold disappears, there is no chance of this late malposition and thus the chance of late and very late thrombosis is absent.

In case of BVS critical observation made by IVUS between 6 -month and 2-year follow-up was a late luminal enlargement (10.9\%) with significant plaque media reduction (12.7\%) and without significant change in the vessel wall area (EEM). Still today, it is unknown whether this 'plaque media regression' on IVUS is a true atherosclerotic regression, with change in vessel wall composition and plaque morphology (from thin-cap atheroma to thick-cap atheroma) or a pseudo-regression due to bioresorption of the polymeric struts. ${ }^{21}$

\section{Imaging of BVS (ABSORB stent)}

BVS is a radiolucent stent. So under fluoroscopy they are detected by the presence to two platinum markers on either side. The ultrasonic and light wave beams from the gray-scale intravascular ultrasound (GS-IVUS) and OCT catheters are backscattered in different ways from the polymeric structures as compared with metallic structures. Consequently, GS-IVUS and OCT render the BVS structures differently compared with metallic stents.

GS-IVUS imaging, with a $20 \mathrm{MHz}$ catheter, renders the polymeric struts as hyper-refractive boxes with an important echogenic blooming effect that confers a double strut appearance. The polymeric struts have an echogenic intensity similar to calcium tissue, but without acoustic shadowing behind the struts (Fig. 5). OCT shows the polymeric struts as a black central core surrounded by a light-scattering frame. The four sides of the polymeric struts are clearly visible without the typical shadowing observed behind metallic structures. In a substudy of ABSORB cohort B trial involving 48 patients, it was shown that GS-IVUS has a poor capacity to detect qualitative findings post-BVS implantation and its reproducibility is low compared with OCT. The use of GS-IVUS should be limited when assessing lumen and scaffold areas. ${ }^{22}$ 

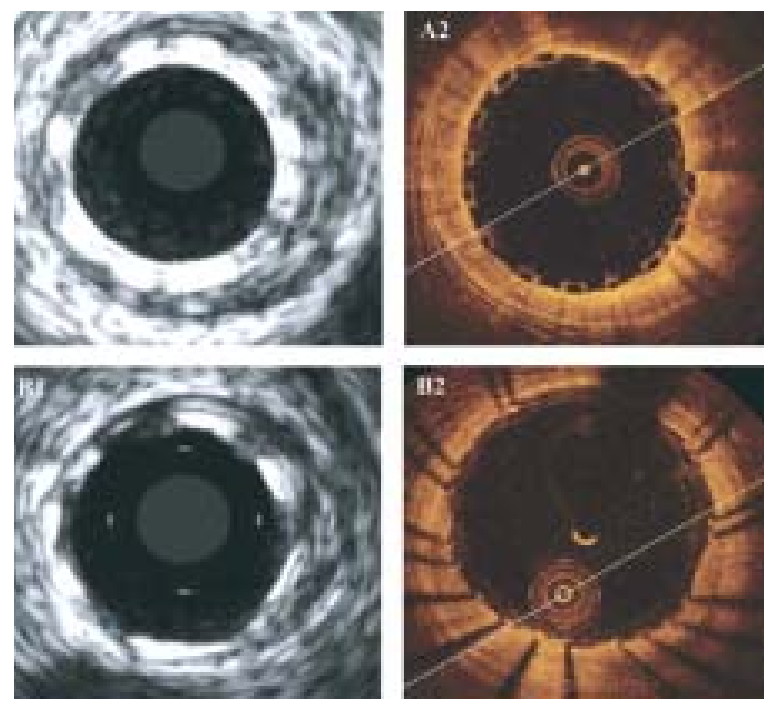

Fig.-5: GS-IVUS and OCT imaging of the bioresorbable scaffolds and metallic stents (A1) GS-IVUS image of a BVS; (A2) OCT image of a BVS; (B1) GS-IVUS image of a metallic stent;

(B2) OCT image of a metallic stent. ${ }^{22}$

(Reproduced with permission.)

\section{Who are candidates for BVS}

Regarding the lesions in which BVS (ABSORB stent) can be used has got some limitation. Safety and efficacy of the stent has been proved in patients aged more than 18 years with stable and unstable angina by ABSORB cohort B trial. Acute myocardial infarction or unstable arrhythmias, left ventricular ejection fraction less than $30 \%$, restenotic lesions, lesions located in the left main coronary artery,ostial lesions, lesions involving a side branch more than $2 \mathrm{~mm}$ in diameter, and lesions with intracoronary thrombus, heavy calcification, excessive tortuosity or other clinically significant stenoses in the target vessel were excluded from the ABSORB cohort A, ABSORB cohort B, ABSORB EXTEND, ABSORB II trial. So the safety and efficacy of the BVS in these lesions is not proven yet. Two de novo native coronary artery lesions each located in different epicardial vessels were also subjected to ABSORB stent and proved safe.

At present, ABSORB stent is the only BVS available for use. But it is available in only few limited sizes [diameter $3.5 \mathrm{~mm}$ (length $28 \mathrm{~mm}$ ), $3.0 \mathrm{~mm}$ (length 12, 18 and $28 \mathrm{~mm}$ ), $2.5 \mathrm{~mm}$ (length 12, 18,28 mm)]. So BVS can be used only in limited number of lesions which matches the size of the available stents. Under-sizing of the $3.0 \mathrm{~mm}$ device in small vessels $(2.5 \mathrm{~mm})$ has been shown to be potentially not of concern based on preliminary data from the first 52 patients in ABSORB Cohort B, as assessed by 6 -month angiographic, IVUS, and clinical outcomes. ${ }^{23} \mathrm{It}$ is because of the fact that the $2.5 \mathrm{~mm}$ ABSORB is actually the same device as the $3.0 \mathrm{~mm}$ device but crimped onto a smaller balloon, the deployment of a $3.0 \mathrm{~mm}$ ABSORB in small vessels $(<2.5 \mathrm{~mm})$ did not compromise safety. But implantation of ABSORB stent in an oversized vessel will lead to strut fracture and spontaneous Incomplete Stent Apposition (ISA) immediately after deployment, which may lead to stent thrombosis.

\section{Technical aspects of BVS (ABSORB) implantation-}

At present ABSORB BVS 1.1 is available in limited number of sizes. So appropriate sizing is mandatory. In ABSORB cohort B and ABSORB EXTEND Single Arm Study, vessel diameter was assessed by QCA and IVUS. Maximum diameter of the vessel measured by QCA proximal and distal to the lesion helped in appropriate sizing of the stents. ${ }^{24}$ In ABSORB II trial stents with $2.5 \mathrm{~mm}$ diameter was used for the Dmax $>2.25$ $\mathrm{mm}$ to d" $3.0 \mathrm{~mm}$; $3.0 \mathrm{~mm}$ diameter stents for e" 2.5 to d" $3.3 \mathrm{~mm}$ and $3.5 \mathrm{~mm}$ stents for e" 3.0 to $<3.8 \mathrm{~mm}$.

Pre-dilatation prior to BVS implantation is mandatory. Post-dilatation with a balloon shorter than the implanted scaffold is at the operator's discretion. But overinflation has got a chance of strut fracture. As the ABSORB stents are radiolucent the stent should be detected by the platinum markers located on either side of the stent. Appropriateness of implantation should be evaluated by OCT.

All patients should be pre-treated with aspirin and a loading dose of at least $300 \mathrm{mg}$ of clopidogrel. Post-procedurally, all patients should receive aspirin $75 \mathrm{mg}$ lifelong and clopidogrel 75 $\mathrm{mg}$ daily for a minimum of 6 months. ${ }^{24}$

\section{Long term trials with BVS}

In a single-arm, prospective, open-label study (ABSORB cohort A), with safety and imaging 
endpoints, 30 patients were enrolled at four participating sites between March and July 2006. ABSORB 1.0 stent was used in this trial. Patients were older than 18 years, with a diagnosis of stable, unstable or silent ischaemia. All treated lesions were single with a percent diameter stenosis e" $50 \%$ and $<100 \%$ with a thrombolysis in myocardial infarction (TIMI) flow grade of e"1. Major exclusion criteria were patients presenting with an acute myocardial infarction, unstable arrhythmias or patients who had left ventricular ejection fraction $<30 \%$, restenotic lesions, lesions located in the left main coronary artery, lesions involving a side branch $>2 \mathrm{~mm}$ in diameter, and the presence of thrombus or another clinically significant stenosis in the target vessel. Clinical endpoints were assessed at 30 days, six and nine months, at one, two and three years. One patient underwent nonischaemia driven target lesion revascularization (TLR) and two patients underwent nonischaemia driven target vessel revascularization (TVR) within first 4 months. Overall, there were no new MACE events between six months and three years. In the entire cohort, there was no instance of stent thrombosis according to either the protocol or ARC definitions. ${ }^{25}$ Five-year clinical follow-up is available for 29 patients. At 5 years, the ischemia-driven MACE rate of $3.4 \%$ remained unchanged. ${ }^{26}$

In ABSORB cohort B trial 101 patients were included and followed up for three years. ABSORB 1.1 stents were used in this trial. During the three-year follow-up period, there were no possible, probable, or definite scaffold thromboses. There were three non-Q-wave myocardial infarctions and seven ischaemia driven-TLRs, which resulted in a three-year MACE rate of $10 \%$. QCA was done at $6,12,24$ and 36 months. There were six in-segment restenoses at three years with a binary restenosis rate of $6 \% .{ }^{27}$

A non-randomized comparison of clinical outcomes of the Absorb BVS scaffold and the XIENCE V metallic stent was done involving the patients in ABSORB and SPIRIT trial. It shows that these two devices have possibly similar event rates up to three years. ${ }^{27}$

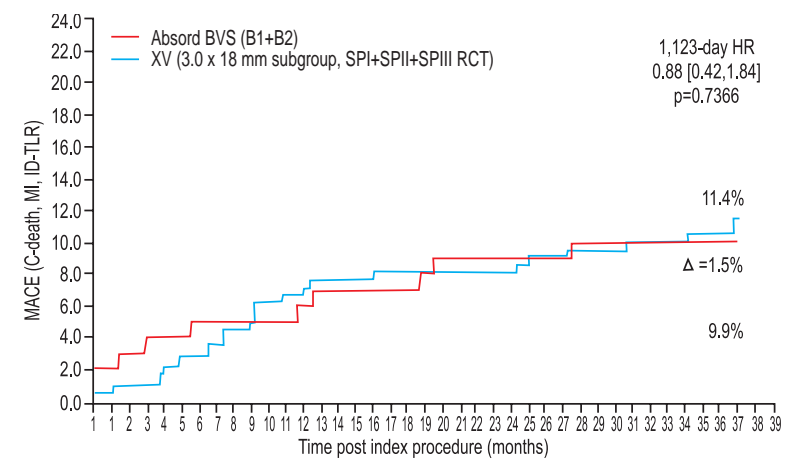

Fig.-6: Kaplan-Meier estimates of cumulative major adverse cardiac events (cardiac mortality, any myocardial infarction or ischaemiadriven target lesion revascularisation) of the ABSORB cohort $B$ (red, $n=101)$ and the 226 patients who received a single $3.0 \times 18 \mathrm{~mm}$ metallic everolimuseluting stent in the SPIRIT I, II and III trials (blue). ${ }^{27}$ (Reproduced with permission.)

The assessment of 'real world' treatment of de novo coronary artery disease with the Revision 1.1 BVS has begun in the ABSORB EXTEND multinational continued access trial, and interim data for the first 585 patients enrolled were presented. Of the first 500 patients there was a $97 \%$ procedural success, $0.6 \%$ TLR and $3.0 \%$ MACE rate at 6 months. ${ }^{28}$ Furthermore, 250 patients have 12 months' follow-up clinical data with a $2.0 \%$ TLR and $4.4 \%$ MACE rate (comparable to the pooled SPIRIT trials event rate of $6.1 \%){ }^{29}$

The ABSORB II trial is planned to be powered for non-inferiority versus the Xience V DES. Patient characteristics and angiographic characteristics are similar to the previous trials, except lesions upto $48 \mathrm{~mm}$ length will be included in the study. Lesions with proximal or distal Dmax of 2.25 to $3.8 \mathrm{~mm}$ will be included in the study. ABSORB BVS 1.1 stents with 2.5, 3.0 and $3.5 \mathrm{~mm}$ diameter will be used. Exclusion criteria are same like the previous trials. Clinical follow-up is planned at 30 and 180 days and at 1 , 2 , and 3 years. All subjects will undergo coronary angiography, IVUS and IVUS-virtual histology at baseline (pre-device and post-device implantation) and at 2-year angiographic followup. The primary end point is superiority of the Absorb BVS vs. XIENCE stent in terms of vasomotor reactivity of the treated segment at 2 years. The coprimary end point is the 
noninferiority (reflex to superiority) of the QCAderived minimum lumen diameter at 2 years. ${ }^{30}$

\section{Small side branch occlusion and BVS (ABSORB stent)}

A post hoc analysis of ABSORB Extend trial was done to evaluate the incidence and clinical consequence of small side branch occlusion (SBO). The data from ABSORB Extend was compared with those of SPIRIT trial using Everolimus Eluting Stent (EES). Increased but statistically insignificant amount of postprocedural SBO was observed in BVS group $(6.0 \%$ vs. $4.1 \%$. $\mathrm{p}=0.09$ ). Patients with post-procedural SBO were significantly associated with an increased incidence of in-hospital myocardial infarction. Increased strut thickness was probably responsible for this increased incidence of SBO in BVS. ${ }^{31}$

\section{BVS and myocardial bridge:}

In the ABSORB cohort B2, two patients with a myocardial bridge have been included. One patient had an ischemia driven -TLR at three months. The second patient had aggravation of late loss from one year $(0.96 \mathrm{~mm})$ to three years $(1.22 \mathrm{~mm})$. Authors concluded that it would be prudent to consider myocardial bridging as a contraindication for treatment with a bioresorbable scaffold. ${ }^{27}$

\section{BVS in chronic total occlusion (CTO)}

CTOs were not included in the ABSORB trials. Some operators reported implantation of BVS in CTO lesions, but it needs long term study to evaluate whether it will be effective and safe in CTO lesion. ${ }^{32}$

\section{BVS in small vessel}

In ABSORB cohort B trial 41 patients had vessel diameter of $<2.5 \mathrm{~mm}$ and 60 patients had vessel diameter of e" $2.5 \mathrm{~mm}$. At 2-year angiographic follow-up no differences in late lumen loss, and in-segment binary restenosis were demonstrated between groups. In the small-vessel group, IVUS analysis showed a significant increase in vessel area, scaffold area and lumen area between 6 months and 2-year follow-up. No differences in plaque composition were reported between groups at either time point. At 2-year clinical follow-up, no differences in ischaemia-driven major adverse cardiac events, myocardial infarction or ischaemia-driven target lesion revascularization were reported between small and large vessels. No deaths or scaffold thrombosis were observed. ${ }^{33}$

\section{BVS in calcified lesion}

Unlike metallic stents, these scaffolds have less radial strength and expansion of the vessel by stretching is difficult. Besides this the currently available BVSs have scaffold thickness of $150 \mu$, which is significantly higher than the currently available second- and third-generation DESs, which have the strut thickness of $<95 \mu$. This property makes the BVS relatively high profile and hence adequate lesion preparation is necessary before implantation of BVS in calcified lesions with use of noncompliant scoring balloons and rotational atherectomy. Adequate expansion of stent for perfect strut apposition is also very important. As the BVS are radiolucent, adequate expansion can't be verified by angiography. It needs OCT to ensure appropriate stent implantation. Whether use of BVS in calcified lesions will be appropriate or not and the technical aspects of such use is not yet decided by any trial. ${ }^{34}$

BVS in ST-elevation myocardial infarction The use of BVS in ST elevation myocardial infarction (STEMI) has not yet been validated by any clinical trial. KocÇka et al reported two cases of BVS use in STEMI without any inhospital adverse outcome. The authors believe that primary PCI in the STEMI setting might represent a suitable BVS indication. Patients with STEMI tend to be younger and any possible benefits of BVS are more likely to become evident with longer term follow-up. Another case report was done by Jaguszewski et al about the use of BVS in STEMI. But it was complicated by acute thrombosis of the stent. OCT was done later, which revealed incomplete apposition of the struts. It seems prudent to ensure that every BVS implanted in highly thrombotic milieu is optimally expanded and some future trial will evaluate the use of BVS in STEMI and thrombotic lesions. ${ }^{35,36}$

\section{Overlapping BVS stents}

ABSORB stents are available in three different sizes 12,18 and $28 \mathrm{~mm}$. In real time practice long 
lesions are to be dealt. Besides this when an edge dissection develops a second stent is always needed. In ABSORB trials long lesions were avoided, though in ABSORB extend SAS trial up to $28 \mathrm{~mm}$ lesions and in ABSORB II up to $48 \mathrm{~mm}$ lesions are being dealt. So the experience with overlapping of the ABSORB stents is still waiting. Some individual case reports of using overlapping have been reported. The major difficulty is the strut size $(150 \mu)$ of the stent. The delivery technique of two high profile stents will be difficult. The absorption kinetic and drug elution technique in case of overlapping stents is not well established. We have to wait for future trials to establish the fact. Some better design with a more low profile stent might allow us to deal this sort of complex lesions. ${ }^{37}$ A study involving 41 overlapping ABSORB stents in pig has demonstrated that overlapping struts gives rise more neointimal response. At 28 days strut coverage was $79 \%$ in case of ABSORB and $99.6 \%$ in case Xience V. But at 90 days it was $>99 \%$ in both the stent groups. Increased strut thickness was probably responsible for this delayed strut coverage. ${ }^{38}$

\section{Absorbable metallic stent:}

Absorbable metal stents (AMS) were designed to overcome drawbacks of BMS and DES. The idea was that AMS will provide the scaffolding support to the coronary arteries for the initial period of time after PCI, and then it will disappear. It will also be superior to PLLA scaffolds by providing better apposition to the vessel wall and can also be used in complex lesions like calcified lesions and chronic total occlusions. Two different metals have been triedi) magnesium alloy, ii) stainless steel. Stainless steel takes about 4 years to absorb and it was not tried that much.

\section{Biodegradable magnesium stent:}

The first metallic bioabsorbable stent implanted in humans is the magnesium alloy stent. It is balloon expandable, with strut thickness of 165 $\mu \mathrm{m}$, and the $3-\mathrm{mm}$ stent has a crossing profile of $1.2 \mathrm{~mm}$ compatible with a $6 \mathrm{~F}$ guide catheter. The coverage of arterial wall by the expanded stent is similar to that of the conventional metallic stents $(10 \%) .{ }^{39}$ The radial strength at implantation is similar to that of stainless steel stents. ${ }^{10}$ The stent is radiolucent.

The PROGRESS-AMS was a prospective, multicenter clinical trial of 63 patients with coronary artery disease who underwent PCI with Absorbable Magnesium Stent (AMS) implantation. This study demonstrated that biodegradable magnesium stents can be implanted safely and that the stents degraded as intended without stent thrombosis, myocardial infarction, or death at 1 year. ${ }^{40}$ However, the study was associated with high angiographic restenosis rates of in-stent diameter stenosis (DS) $(48.2 \pm 17.0 \%)$ and insegment late loss $(0.83 \pm 0.51 \mathrm{~mm})$, which led to ischemic-driven target lesion revascularization of $23.8 \%$ at 4 months and $27.9 \%$ at 1 year. Intravascular ultrasound imaging supported the degradation of AMS at 4 months. They concluded that slower degradation is needed to provide sufficient radial force to improve long-term patency rates of the AMS. ${ }^{41}$

To overcome these drawbacks a drug-eluting absorbable metal scaffold (DREAMS; Biotronik, Bülach, Switzerland) is being tried. DREAMS was optimized to provide a longer scaffolding time than its predecessor and drug elution for inhibition of cell proliferation. The absorption process is complete after 9-12 months. The scaffold strut surface is coated with a $1 \frac{1 / 4}{\mathrm{~m}}$ matrix of the absorbable polymer carrier polylactic-coglycolic acid and the antiproliferative drug paclitaxel. ${ }^{42}$

BIOSOLVE-I was a prospective, non-randomized, multicentre, first-in-man trial to assess the safety and performance of a paclitaxel-eluting absorbable magnesium scaffold (DREAMS) in symptomatic patients with de-novo coronary lesions. The study was done at 5 centers in Europe. The study included 46 patients with 47 lesions. 12 patients had Type I lesion, 31 had Type IIa lesions and 4 had Type IIb lesions. Seven patients had moderate to severely calcified lesions. Patients were followed up for 36 months. At 12 months, 3 patients developed target lesion failure (7\%). In-scaffold late lumen loss was 0.65 $\mathrm{mm}$ at 6 months and $0.52 \mathrm{~mm}$ at 12 months. It was better than AMS in PROGRESS study, but inferior to DES and ABSORB stent. 
Implantation of the bioabsorbable everolimus eluting coronary scaffold system necessitated bailout stenting in $5 \cdot 3 \%$ of the lesions, but DREAMS had a $100 \%$ procedural and device success rate. They concluded that the results of BIOSOLVE - 1 with DREAM stent were noninferior to those with DES and ABSORB stents in most aspects. But further iterations of the device will need to be designed to reduce late lumen loss. ${ }^{43}$

\section{The Igaki-Tamai stent}

The Igaki-Tamai stent (Igaki Medical Planning Company, Kyoto, Japan), the first absorbable stent implanted in humans, is constructed from PLLA. Strut thickness is larger than that in contemporary metallic stents at $170 \mu \mathrm{m}$, and vessel coverage by stent struts (24\%) is greater than that with contemporary metallic stents. This balloon-mounted self-expanding sheathed system where expansion is hastened by dilatation with warmed contrast medium $\left(80^{\circ} \mathrm{C}\right)$ is cumbersome to use. As PLLA is radiolucent, gold markers at each end provide radio-opacity for stent identification. The stent does not release an antiproliferative drug. Stent struts retain shape until absorption is well advanced. In the preliminary, first-in-man prospective, nonrandomized clinical trial that enrolled 50 patients, a 4-year follow-up of all the patients $(100 \%)$ revealed a low complication rate with one in-hospital stent thrombosis causing a Q-wave myocardial infarction, one noncardiac death, and $18 \%$ repeat $\mathrm{PCI}$ and no surgical revascularization. ${ }^{4}$

Nishio et al report beyond 10 years' clinical outcome of the first 50 patients with coronary artery disease who were treated with 84 IgakiTamai stents. Restenosis rates are similar to those reported with bare metal stents. But the artery restored its capability to respond to positive remodeling once the scaffold degraded. ${ }^{44}$

\section{Newer PLLA based stents}

DeSolve (Elixir Medical Corporation, Sunnyvale,California) is a new PLLA based stent with strut thickness of $150 \mu \mathrm{m}$. The scaffold is coated with a matrix of polylactide-based polymer and myolimus at a $3 \mathrm{mg} / \mathrm{mm}$ dose; more than $85 \%$ of the drug is released over 4 weeks. The system has a crossing profile of $1.47 \mathrm{~mm}$ and is $6-\mathrm{F}$ catheter compatible. This new device provides good radial strength for 4 months. And complete resorption of the scaffold occurs by 1 year. It has got the ability to self-correct to the vessel wall in cases of minor malapposition when expanded to the nominal diameter, which is accomplished using a proprietary processing technique. DeSolve stent has a wide safety margin for expansion where a 3.0-mm scaffold can be expanded to $4.5 \mathrm{~mm}$ in diameter without strut fracture.

A human trial using DeSolve stent was done in 16 patients showing good short and midterm results (upto 12 months) similar to ABSORB stents. The advantage of DeSolve stent over ABSORB stent is its early resorption without hampering the early or late lumen loss. So there is early anatomical and physiological normalization of blood vessels. Its ability to selfcorrect to the vessel wall in cases of early malapposition also gives an extra advantage. ${ }^{45}$

\section{The REVA device}

The REVA bioabsorbable scaffold is a tyrosinederived polycarbonate polymer device impregnated with iodine for radio-opacity. Initial FIM clinical trials $(n=27)$ reported high clinical event rates (TLR 67\%) attributed to focal mechanical failures of the device. ${ }^{46}$ This device has been redesigned, resulting in the new ReZolve device (with a stronger polymer, sirolimus drug coating, and a novel slide and spiral lock mechanism). Clinical evaluation of this device began in December 2011 (RESTORE trial) and preliminary safety and performance data demonstrated $100 \%$ clinical procedural success rate and zero MACE events at 3 months $(n=22){ }^{47}$ However, this device has a sheathed delivery mechanism, limiting its use in small or tortuous vessels. Therefore, further development has resulted in the new ReZolve2 device, which has a lower prolife $(6 \mathrm{~F})$, and a sheathless delivery system with an enhanced polymer, yielding a $30 \%$ 
increase in radial strength. The RESTORE study has been amended to allow the use of this next generation device, as will the RESTORE II multicentre global trial. ${ }^{48}$

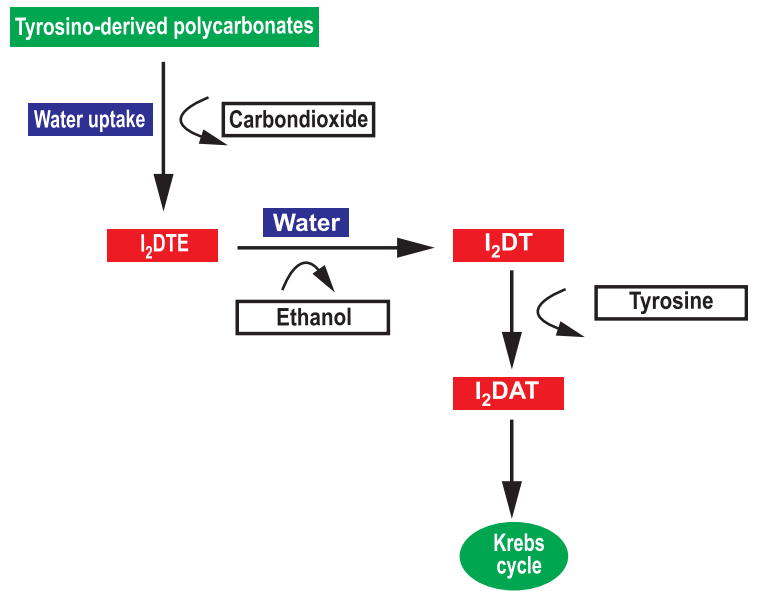

Fig.-7: The Metabolism of Tyrosine-Polycarbonate Stents Initially, hydrolysis of the tyrosinepolycarbonate produces iodinated desaminotyrosyl-tyrosine ethyl esters (I2DTE), and releases carbon dioxide. I2DTE is hydrolyzed into iodinated desaminotyrosyl-tyrosine (I2DT) and ethanol. Cleavage of I2DT produces tyrosine and iodinated desaminotyrosine (I2DAT), which enters the Krebs cycle. ${ }^{48}$ (Reproduced with permission.)

\section{The IDEAL device}

This device has a polylactide anhydrite and salicylic acid-sebacic acid-salicylic acid trimer polymer backbone providing mechanical scaffolding, and a salicylic acid-adipic acidsalicylic acid trimer with sirolimus coating layer. The additional elution of salicylic acid has been shown to reduce inflammation in animal studies and may infer an advantage over 'conventional' polymers. The safety of the first generation IDEAL device (Bioabsorbable Technology, California, USA) was confirmed in the Whisper trial $(n=11)$ with no early recoil. However, there was significant neointimal growth.

The second generation IDEAL BioStent (Xenogenics Corp, Massachusetts, USA) has an optimized scaffold design, higher drug dosage, slower drug release and alternations in delivery mechanisms conferring a reduction to $6 \mathrm{~F}$ compatibility. Preclinical data are awaited for this iteration. ${ }^{49}$

\section{Future of BVS}

DES is a major breakthrough in interventional cardiology because they reduced the need for repeat intervention compared with BMS by more than $50 \%$. Late (6 month to 1 year) or very late (beyond 1 year) thrombosis is the feared complication of DES that may result in myocardial infarction and death. A worst case scenario is that the problem of late-stent thrombosis after first-generation DES implantation may be ongoing indefinitely as a registry of 8000 patients reported that it

Comparison with conventional stents

\begin{tabular}{lllll}
\hline Stent/Scaffold & Igaki-Tamai & ABSORB & DREAMS & XIENCE-V \\
\hline Polymer/Alloy & PLLA & PLLA & Magnesium & Cobalt chromium \\
Polymer/drug & None & PDLLA/Everolimus & PLGA/Paclitaxel & Fluropolymer/Everolimus \\
Strut thickness, $\mu \mathrm{m}$ & 170 & 150 & 150 & 86 \\
Total absorption, yrs & $3-4$ & 4 & 1 & None \\
Drug dose & None & $100 \mu \mathrm{g} / \mathrm{cm}^{2}$ & $8 \mu \mathrm{g}$ & $100 \mu \mathrm{g} / \mathrm{cm}^{2}$ \\
In stent/scaffold late & 0.91 & ABSORB A 0.44 & 0.64 & 0.10 \\
loss, 6 mon, mm & & ABSORB B 0.19 & & \\
TLR at 6 mon, $\%$ & 10.5 at 6 mon & ABSORB A 3.3 & 4.3 & 3.8 \\
& 26 at 10 yr & ABSORB B 3.6 & & \\
Duration radial support & 6 months & Cohort A-weeks & Days to weeks & Permanent \\
Stent to artery coverage & $24 \%$ & Cohort B-3 months & & 10.7 \\
Crossing profile & $1.5 \mathrm{~mm}$ & $25 \%$ & $10 \%$ & 1.085 \\
\hline
\end{tabular}


occurred at a constant rate of $0.6 \%$ per annum without diminution by 4 years. ${ }^{50}$ Indefinite continuation of dual antiplatelet therapy is not a practical solution because of expense and because a quarter of late-stent thromboses occurred in patients receiving dual antiplatelet therapy. ${ }^{52}$ In addition, dual antiplatelet therapy may be discontinued because of troublesome minor bleeding or to reduce the risk of bleeding during surgery.

An ideal stent will be one which will give the scaffold action for the appropriate time period, will carry antiproliferative drug to prevent neointimal growth and will disappear thereafter. Bioabsorbable drug eluting stents are a great hope as this group of ideal stents.

Despite its initial promise, BVS technology has major challenges. By its nature, the plastic PLLA polymer is limited in expansion and optimal scaffold apposition. Overexpansion of the scaffold may result in fractures that can lead to target vessel failure. Furthermore, it is not clear how the PLLA scaffold will behave in calcified lesions, bifurcations, long lesions, or when overlapping of additional PLLA scaffolds is required.

In addition, the optimal duration of dualantiplatelet therapy with PLLA is unknown, but complete degradation duration of 3 years raises the question regarding the duration of dualantiplatelet therapy. Finally, manufacturing the PLLA BVS is complex, and the availability of a broad spectrum of vessel sizes and lesion lengths is currently limited. The question is whether PLLA limitations and the additional cost to patients will prevent the ABSORB device from becoming a workhorse in the treatment of coronary artery disease and ultimately replace permanent metal DES.

If an appropriate BVS is available along with the favorable answers to the above questions, it will be able to replace conventional metallic stents. It will reduce late and very late stent thrombosis, so there will be reduced use of antiplatelet agents. Physiologically, the absence of a rigid metallic cage will facilitate the restoration of the vessel vasomotor tone, adaptive shear stress, late luminal enlargement, and late expansive remodeling. In the long term, BVS will not hamper future treatment options such as PCI, CABG, or pharmacologically induced plaque regression. Furthermore, BVS will obviate some of the other problems associated with the use of permanent metallic stents such as the covering of side branches. It will also appear to be suitable for non-invasive imaging such as computed tomographic angiography or magnetic resonance imaging, due to the absence of artifact caused by permanent metallic materials.

\section{Conclusion:}

Potential advantage of having the stent disappear from the treated site is that it will change a diseased coronary artery to normal or near normal artery. Bioabsorbable stents have a potential pediatric role because they allow vessel growth and do not need eventual surgical removal. Considerable improvement has been done in the technology of Biodegradable stents. In case of Magnesium stents further improvement is needed to reduce the late lumen loss. For the PLLA stents, efforts needed to optimize scaffold radial force and expansion without early fractures. Trials should be carried out to define the role of biodegradable stents in more complex lesions like long lesions, calcified lesions, bifurcation lesions, left main lesions, ostial lesions etc. Also its efficacy should be verified in different clinical situations. How does it behave in overlapping conditions is also a concern. How the restenotic lesions of biodegradable stents should be managed is yet to be learned. And of course we should know how long the antiplatelets should be prescribed. Cost of these stents is also a concern. If we get the answers of these questions in a favorable way, only then we will be able to replace the BMS and DES with biodegradable stents. May be at that time we will look back and laugh at the days when we left a permanent metal stent in our patients' coronary arteries.

\section{Conflict of Interest - None.}

\section{References:}

1. Fischman D, Leon M, Baim D, et al. A randomized comparison of coronary stent placement and balloon 
angioplasty in the treatment of coronary artery disease. The Stress Trial. N Engl J Med 1994;331:496-501.

2. Konig A, Schiele TM, Rieber J, et al. Influence of stent design and deployment technique on neointima formation and vascular remodeling. Z Kardiol 2002;91(Suppl 3): 98-102.

3. Serruys PW, Luijten HE, Beatt KJ, et al. Incidence of restenosis after successful coronary angioplasty: a timerelated phenomenon. A quantitative angiographic study in 342 consecutive patients at 1, 2, 3, and 4 months. Circulation 1988;77:361-371.

4. Tamai H, Igaki K, Kyo E, et al. Initial and 6-month results of biodegradable poly-1-lactic acid coronary stents in humans. Circulation 2000;102:399-404.

5. Nishio S, Kosuga K, Okada M, et al. Long term (.10 years) clinical outcomes of the first-in-man biodegradable polyL-lactic acid coronary stents. Oral presentation. Paris: EuroPCR; 2010.

6. Erbel R, Di Mario C, Bartunek J, et al. Temporary scaffolding of coronary arteries with bioabsorbable magnesium stents: a prospective, non-randomised multicentre trial. Lancet 2007;369:1869-1875.

7. Pollman M. Engineering a bioresorbable stent: the REVA programme update. Eurointervention 2009;5(Suppl. F):F54-F57.

8. Aalst M, Eenink M, Kruft M, van Tuil R. ABC's of bioabsorption: application of lactide based polymers in fully resorbable cardiovascular stents. Eurointervention 2009;5(Suppl. F):F23-F27.

9. Garg S, Serruys PW. Coronary Stents -Looking Forward. J Am Coll Cardiol 2010;56:S43-78.

10. Okamura T, Garg S, Gutierrez-Chico JL, et al. In vivo evalution of stent strut distribution patterns in the bioabsorbable everolimus-eluting device: an OCT ad hoc analysis of the revision 1.0 and revision 1.1 stent design in the ABSORB clinical trial. EuroIntervention 2010;5:932-938.

11. Lara JG, Brugaletta S, Diletti R, et al. A comparative assessment by optical coherence tomography of the performance of the first and second generation of the everolimus-eluting bioresorbable vascular scaffolds. Eur Heart J 2011; 32: 294-304.

12. Serruys PW, Onuma Y, Ormiston JA, et al. Evaluation of the Second Generation of a Bioresorbable Everolimus Drug-Eluting Vascular Scaffold for Treatment of De Novo Coronary Artery Stenosis - Six-Month Clinical and Imaging Outcomes. Circulation 2010;122:2301-2312.

13. Surovtsova I. Effects of compliance mismatch on blood flow in an artery with endovascular prosthesis. J Biomech 2005; 38: 2078-2086.

14. Tortoriello A, Pedrizzetti G. Flow-tissue interaction with compliance mismatch in a model stented artery. J Biomech 2004; 37: 1 - 11 .

15. Wentzel JJ, Whelan DM, van der Giessen WJ, et al. Coronary stent implantation changes 3 -D vessel geometry and 3-D shear stress distribution. J Biomech 2000; 33: $1287-1295$.
16. Brugaletta S, Gogas BD, Garcia-Garcia HM, et al. Vascular compliance changes of the coronary vessel wall after bioresorbable vascular scaffold implantation in the treated and adjacent segments. Circ J 2012; 76: 1616 - 1623.

17. Wentzel JJ, Whelan DM, van der Giessen WJ, et al. Coronary stent implantation changes 3 -D vessel geometry and 3-D shear stress distribution. J Biomech 2000;33: 1287-1295.

18. Gomez-Lara J, Garcia-Garcia HM, Onuma Y, et al. A comparison of the conformability of everolimus-eluting bioresorbable vascular scaffolds to metal platform coronary stents. J Am Coll Cardiol Intv 2010;3:1190-1198.

19. Gomez-Lara J, Brugaletta S, Farooq V, et al. Angiographic geometric changes of the lumen arterial wall after bioresorbable vascular scaffolds and metallic platform stents at 1-year follow-up. J Am Coll Cardiol Intv 2011;4:789-799.

20. Cook S, Ladich E, Nakazawa G, et al. Correlation of intravascular ultrasound findings with histopathological analysis of thrombus aspirates in patients with very late drug-eluting stent thrombosis. Circulation 2009;120: 391-399.

21. Sarno G, Onuma Y, Garcia Garcia HM, et al. IVUS radiofrequency analysis in the evaluation of the polymeric struts of the bioabsorbable everolimus-eluting device during the bioabsorption process. Catheter Cardiovasc Interv 2010;75: 914-918.

22. Go'mez-Lara J, Brugaletta S, Diletti R, et al. Agreement and Reproducibility of Gray-Scale Intravascular Ultrasound and Optical Coherence Tomography for the Analysis of the Bioresorbable Vascular Scaffold. Catheter Cardiovasc Interv 2012; 79:890-902.

23. Diletti R, Onuma Y, Farooq V, et al. Clinical and intravascular imaging outcomes at 1 and 2 years after implantation of absorb everolimus eluting bioresorbable vascular scaffolds in small vessels. Late lumen enlargement: does bioresorption matter with small vessel size? Insight from the ABSORB cohort B trial. Heart 2013;99:98-105.

24. Farooq V, Gomez-Lara J, Brugaletta S, et al. Proximal and distal maximal luminal diameters as a guide to appropriate deployment of the ABSORB Everolimuseluting Bioresorbable Vascular Scaffold: A sub-study of the ABSORB Cohort B and the on-going ABSORB EXTEND Single Arm Study. Catheter Cardiovasc Interv 2012; 79:880-888.

25. Onuma Y, Serruys PW, Ormiston JA, et al. Three-year results of clinical follow-up after a bioresorbable everolimus-eluting scaffold in patients with de novo coronary artery disease: the ABSORB trial. EuroIntervention 2010;6:447-453.

26. Onuma Y, Dudek D, Thuesen L, et al. Five-year clinical and functional multislice computed tomography angiographic results after coronary implantation of the fully resorbable polymeric everolimus-eluting scaffold in patients with de novo coronary artery disease. The ABSORB Cohort A Trial. J Am Coll Cardiol Intv 2013;6:999-1009. 
27. Serruys PW, Onuma Y, Garcia-Garcia HM, et al. Dynamics of vessel wall changes following the implantation of the Absorb everolimus-eluting bioresorbable vascular scaffold: a multi-imaging modality study at $6,12,24$ and 36 months. Eurointervention 2013 Dec 3. pii: 2013/0/7e. [Epub ahead of print].

28. Bartorelli AL. ABSORB EXTEND: An Interim Report on the 6 -month clinical outcome from the first 500 patients registered. Presented at TCT 2012.

29. Bartorelli AL. ABSORB EXTEND: An Interim Report on the 12-Month Clinical Outcomes from the First 250 Patients Registered. Presented at TCT 2012.

30. Diletti R, Serruys PW, Farooq V, et al. ABSORB II randomized controlled trial. A clinical evaluation to compare the safety, efficacy, and performance of the Absorb everolimus-eluting bioresorbable vascular scaffold system against the XIENCE everolimus-eluting coronary stent system in the treatment of subjects with ischemic heart disease caused by de novo native coronary artery lesions: Rationale and study design. Am Heart J 2012;164:654-663.

31. Muramatsu T, Onuma Y, Garc1'a-Garc1'a HM, et al, on behalf of the ABSORB-EXTEND Investigators. Incidence and short-term clinical outcomes of small side branch occlusion after implantation of an everolimus-eluting bioresorbable vascular scaffold. An interim report of 435 patients in the ABSORB-EXTEND Single-Arm Trial in comparison with an everolimus-eluting metallic stent in the SPIRIT First and II Trials. J Am Coll Cardiol Intv 2013;6: 247-257.

32. Manna AL, Ohno Y, Attizzani GF, Tamburino C. Successful retrograde recanalization of chronic total coronary occlusion with multiple bioresorbable vascular scaffolds ('full polymer jacket'): initial experience and rationale. Eur Heart J 2013; 34(37): 2925.

33. Diletti R, Farooq V, Girasis C, et al. Clinical and intravascular imaging outcomes at 1 and 2 years after implantation of absorb everolimus eluting bioresorbable vascular scaffolds in small vessels. Late lumen enlargement: does bioresorption matter with small vessel size? Insight from the ABSORB cohort B trial. Heart 2013, 99, 98-105.

34. Basavarajaiah S, Naganuma T, Latib A, Colombo A. Can Bioabsorbable Scaffolds Be Used in Calcified Lesions? Cath Cardiovasc Interv 2013. doi: 10.1002/cced24939 [Epub ahead of print].

35. KocÇka V, Lisa L, TousÇek P, BudeÇsÇ1'nsky' T, Widimsky' P. ST elevation myocardial infarction treated with bioresorbable vascular scaffold: rationale and first cases. Eur heart J 2013; 34(27): 2046.

36. Jaguszewski M, Wyss C, Alibegovic J, Lu“ scher TF, Templin C. Acute thrombosis of bioabsorbable scaffold in a patient with acute coronary syndrome. Eur heart J 2013; 34(27): 2073.

37. Meincke F, Kuck KH, Bergmann MW. Delivery of a Bioresorbable Vascular Scaffold to Complex Lesions. Catheter Cardiovasc Interv. doi: 10.1002/ccd.25105.

38. Farooq V, Serruys PW, Heo JH, et al. Intracoronary Optical Coherence Tomography and Histology of Overlapping Everolimus-Eluting Bioresorbable Vascular
Scaffolds in a Porcine Coronary Artery Model The Potential Implications for Clinical Practice. J Am Coll Cardiol Intv 2013;6:523-532.

39. Ormiston J, Serruys PW, Regar E, et al. A bioabsorbable everolimus-eluting coronary stent system for patients with single de-novo coronary artery lesions (ABSORB): a prospective open-label trial. Lancet 2008;371:899-907.

40. Erbel R, Di Mario C, Bartunek J, et al., PROGRESS-AMS (Clinical Performance and Angiographic Results of Coronary Stenting with Absorbable Metal Stents) Investigators. Temporary scaffolding of coronary arteries with bioabsorbable magnesium stents: a prospective, nonrandomised multicentre trial. Lancet 2007;369:1869-1875.

41. Waksman R, Erbel R, Di Mario C, et al. On behalf of the PROGRESS-AMS (Clinical Performance and Angiographic Results of Coronary Stenting with Absorbable Metal Stents) Investigators. Early- and Long-Term Intravascular Ultrasound and Angiographic Findings After Bioabsorbable Magnesium Stent Implantation in Human Coronary Arteries. J Am Coll Cardiol Intv 2009;2:312-320.

42. Wittchow E, Adden N, Riedmueller J, et al. Bioresorbable drug-eluting magnesium-alloy scaff old: design and feasibility in a porcine coronary model. EuroIntervention 2013; 8(12): 1441-1450.

43. Haude M, Erbel R, Erne P, et al. Safety and performance of the drug-eluting absorbable metal scaff old (DREAMS) in patients with de-novo coronary lesions: 12 month results of the prospective, multicentre, first-in-man BIOSOLVE-I trial. Lancet 2013; 381: 836-844

44. Nishio S, Kosuga K, Igaki K, et al. Long-term (10 years) clinical outcomes of first-in-human biodegradable poly-Llactic acid coronary stents: Igaki-Tamai stents. Circulation 2012;125: 2343-2352.

45. Verheye S, Ormiston JA, Stewart J, et al. A nextgeneration bioresorbable coronary scaffold system from bench to first clinical evaluation six- and 12-month clinical and multimodality imaging results. $\mathrm{J} \mathrm{Am} \mathrm{Coll} \mathrm{Cardiol} \mathrm{Intv}$ 2014; 7(1): 89-99.

46. Pollman MJ. Engineering a bioresorbable stent: REVA programme update. EuroIntervention 2009;5(Suppl F):F54-7.

47. Costa RA. REVA ReZolve Clinical Program Update. Presented at: TCT October 2012.

48. Niket Patel, Adrian P Banning. Bioabsorbable scaffolds for the treatment of obstructive coronary artery disease: the next revolution in coronary intervention? Heart 2013;99:1236-1243.

49. Jabara R, Chronos N, Robinson K. Novel bioabsorbable salicylate-based polymer as a drug-eluting stent coating. Catheter Cardiovasc Interv 2008;72:186-194.

50. Wenaweser P, Daemen J, Zwahlen M, et al. Incidence and correlates of drug-eluting stent thrombosis in routine clinical practice: 4-year results from a large 2 -institutional cohort study. J Am Coll Cardiol 2008;52:1134-1140.

51. Daemen J, Wenaweser P, Tsuchida K, et al. Early and late coronary stent thrombosis of sirolimus-eluting and paclitaxel-eluting stents in routine clinical practice: data from a large two-institutional cohort study. Lancet 2007;369:667-678. 